\title{
The prediction of microvascular invasion of hepatocellular carcinoma using multiple imaging modalities
}

\author{
Hideyuki Tamai \\ Department of Hepatology, Wakayama Rosai Hospital, Wakayama 640-8505, Japan. \\ Correspondence to: Dr. Hideyuki Tamai, Department of Hepatology, Wakayama Rosai Hospital, 93-1 Kinomoto, Wakayama-shi, \\ Wakayama640-8505, Japan. E-mail: hdy-tamai@wakayamah.johas.go.jp
}

How to cite this article: Tamai $\mathrm{H}$. The prediction of microvascular invasion of hepatocellular carcinoma using multiple imaging modalities. Hepatoma Res 2018;4:75. http://dx.doi.org/10.20517/2394-5079.2018.98

Received: 10 Sep 2018 First Decision: 12 Oct 2018 Revised: 20 Nov 2018 Accepted: 27 Nov 2018 Published: 18 Dec 2018

Science Editor: Guang-Wen Cao Copy Editor: Cui Yu Production Editor: Huan-Liang Wu

\begin{abstract}
To implement an adequate treatment strategy for solitary hepatocellular carcinoma (HCC), the prediction of microvascular invasion (MVI) is crucial. Metastatic recurrences after curative treatments can result from occult metastasis derived from invisible MVI. For predicting MVI, poorly differentiated or non-singular nodular HCC with a high risk of MVI should be evaluated by common imaging modalities such as ultrasound, contrast enhanced computed tomography (CECT), or magnetic resonance imaging (MRI). Summarizing these predictabilities in previous reports, the accuracies for predicting MVI were $78 \%$ in contrast enhanced ultrasonography (CEUS), $76 \%-89 \%$ in CECT, and $62 \%-77 \%$ in MRI. Those for predicting poor differentiation were $69 \%-92 \%$ in CEUS, $52 \%-90 \%$ in CECT, and $71 \%-75 \%$ in MRI. Those for predicting non-singular nodular type were $92 \%-95 \%$ in CEUS, $81 \%-89 \%$ in MRI, and $91 \%-93 \%$ in the combination of MRI and CECT. Among common imaging modalities, MRI can provide tissue characterization of the HCC using signal intensity. Gadolinium-ethoxybenzyl diethylenetriamine penta-acetic acid-enhanced MRI including diffusion imaging is the most informative imaging modality to predict MVI. Combination of MRI with other imaging modalities or tumor markers may provide a more accurate predicting for MVI. HCC with a high risk of MVI should be treated as advanced HCC even after curative treatment.
\end{abstract}

Keywords: Hepatocellular carcinoma, microvascular invasion, histologic differentiation, ultrasound, computed tomography, magnetic resonance imaging

\section{INTRODUCTION}

There have been rapid advances in the development of imaging modalities as diagnostic tools in recent years. In hepatocellular carcinoma (HCC), imaging plays a greater part than biopsy in its diagnosis. In addition,

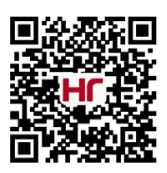


imaging is used not just for its diagnosis, but also for disease surveillance, determination of tumor stage, evaluation of treatment efficacy, and navigation of local treatments. Importantly, imaging has the ability to predict histologic differentiations that reflect the malignant potential of HCC.

As the HCC tumor grows larger, it has a stronger tendency to invade the adjacent portal vein or hepatic vein. HCC with intrahepatic or extrahepatic metastasis derived from vascular invasion is an advanced cancer that is difficult to treat radically ${ }^{[1]}$. However, even if the tumor is solitary on imaging, metastatic recurrences after curative treatments such as surgical resection or local ablation is not uncommon. These metastatic recurrences may result from occult metastasis derived from invisible microvascular invasion (MVI) at the time of diagnostic imaging before treatment was initiated. Therefore, HCC with MVI can be considered as an advanced cancer with occult metastasis. However, there is a limitation in the diagnostic imaging of MVI or occult metastasis. To make an adequate treatment strategy for solitary HCC, the prediction of MVI is crucial. In this review, the present status of the prediction of MVI of HCC using common imaging modalities such as ultrasound (US), computed tomography (CT), and magnetic resonance imaging (MRI) is presented.

\section{FACTORS RELATED TO MVI IN HCC}

HCC develops in a multistep fashion ${ }^{[2]}$. Therefore, most HCCs consist of heterogenously differentiated components. For example, nodule in nodule or mosaic pattern on US imaging represents multistep carcinogene$\mathrm{sis}^{[2]}$. As histologic differentiation advances from well differentiated to poorly differentiated, the prevalence of MVI becomes higher ${ }^{[3]}$. Poor histologic differentiation is a strong predictor of $\mathrm{MVI}^{[4]}$. A large proportion of poorly differentiated HCCs has MVI and intrahepatic metastasis even when the tumor is small ${ }^{[3]}$. As the tumor size increases, a fibrous capsule forms such that a typical HCC is visualized as a nodule within a fibrous capsule. Cancer cell infiltration into the fibrous capsule demonstrates a morphologically invasive feature, and HCCs with infiltrations to the fibrous capsule tend to be poorly differentiated and to have MVI ${ }^{[5]}$. Small nodular HCCs can be macroscopically classified into three types such as single nodular (SN), single nodular with extranodular growth (SNEG), and contiguous multinodular $(\mathrm{CMN})^{[6]}$. Both SNEG and CMN types have a stronger invasive potential, and tend to be more poorly differentiated than the SN type. The prevalence of MVI or microscopic intrahepatic metastasis is also higher in the SNEG and CMN types than in the SN type ${ }^{[7-9]}$. Since MVI is strongly associated with histologic differentiation and the macroscopic type of HCC, the accurate prediction of MVI by imaging will require accurate evaluation of these two parameters.

\section{PREDICTION OF MVI USING US}

US has the highest spatial resolution among common imaging modalities, therefore it can potentially provide an accurate assessment of the macroscopic morphology of HCC. Moribata et al ${ }^{[10]}$ reported the correlation between B mode ultrasonogram and histologic differentiation of small HCC. They revealed that most poorly differentiated small HCCs were visualized as hypoechoic tumor with an irregular or unclear margin on B mode US. However, sensitivity, specificity, positive predictive value (PPV), negative predictive value (NPV), and accuracy of diagnosis for poorly differentiated HCC on the basis of their data are $89 \%, 67 \%, 19 \%$, $99 \%$, and $69 \%$ respectively.

There have been several reports on the prediction of poorly differentiated HCC or MVI using CEUS, based on the evaluation of intra-tumoral angioarchitecture. Sugimoto et al. ${ }^{[1]}$ showed the correlation between the angioarchitecture and histologic differentiation using microflow imaging (MFI) by CEUS. The deadwood pattern of tumoral blood vessels was visualized clearly, but they gradually tapered off and were interrupted suddenly. When HCCs with deadwood pattern were assessed as poorly differentiated, the sensitivity, specificity, positive PPV, NPV, and accuracy of diagnosis for poorly differentiated HCC on the basis of their data are $80 \%, 96 \%, 86 \%, 94 \%$, and $92 \%$, respectively. Tanaka et al. ${ }^{[12]}$ implemented the malignant grading system based on the combined assessment of Kupffer imaging and the maximum intensity projection imaging 
made by the accumulation for each MFI sequence using CEUS with perflubutane microbubbles (Sonazoid; Daiichi-Sankyo, Tokyo, Japan). They classified HCCs into four grades: grade 1 (iso-fine/vascular), grade 2 (hypo-fine), grade 3 (hypovascular), and grade 4 (hypo-irregular). When HCC was assessed as grade 4, sensitivity, specificity, positive PPV, NPV, and accuracy of the diagnosis for poorly differentiated HCC are 100\%, $91 \%, 50 \%, 100 \%$, and $92 \%$ respectively, and those of the diagnosis for MVI are $40 \%, 92 \%, 67 \%, 80 \%$, and $78 \%$ respectively.

The correlation between histologic grading and tumor enhancement washout time on CEUS has also been reported ${ }^{[13-17]}$. For example, Xu et al. ${ }^{[14]}$ analyzed the enhancement pattern of HCC using time-intensity curve. They observed that the time to peak, contrast-enhanced time, and wash-out time of well differentiated HCCs were longer than those of the moderately to poorly differentiated HCCs, whereas the enhancement slope and clearance slope of the well differentiated lesions were lower than those of the moderately to poorly differentiated lesions. However, Pei et al. ${ }^{[15]}$ reported that washout time is the only significant factor (among all the time-intensity curve parameters) correlated to histologic grading. Washout time in the well-, moderately-, and poorly differentiated HCCs was $36.66 \pm 9.61,19.37 \pm 2.83$, and $11.61 \pm 2.78 \mathrm{~s}$, respectively. Feng et al. ${ }^{[17]} \mathrm{re}-$ ported using the washout rate to predict HCC differentiation. They demonstrated that when the cutoff point was set at washout before $40 \mathrm{~s}$ from contrast injection, the ability to distinguish poorly differentiated from moderately- and well differentiated HCCs could be performed with a sensitivity, specificity, and area under the curve (AUC) of $24 \%, 97 \%$, and 0.68 , respectively.

Additionally, several reports have demonstrated the correlation between macroscopic HCC type and Kupffer imaging (post vascular phase) using Sonazoid CEUS. Hatanaka et al ${ }^{[18]}$ reported that the sensitivity, specificity, PPV, NPV, and accuracy of CEUS for predicting for the non-SN type were $80 \%, 96 \%, 92 \%, 89 \%$ and $90 \%$, respectively. Tada et al ${ }^{[19]}$ also reported that those in small HCCs ( $3 \mathrm{~cm}$ or less) were $87 \%, 93 \%, 91 \%, 84 \%$ and $94 \%$, respectively. Furthermore, Hatanaka et al. ${ }^{[20]}$ demonstrated that CEUS was more accurate at distinguishing macroscopic type than contrast CT. This could be explained by the difficulty in evaluating the shapes of nodules on contrast CT because of the partial volume effect. Nuta et al. ${ }^{[21]}$ also indicated that HCCs with an irregular defect visualized during Kupffer-phase of CEUS were characterized by more frequent MVI and intrahepatic metastasis. They demonstrated that Kupffer-phase images were more accurate at predicting the macroscopic pathologic type with high grade malignancy (SNEG or CMN type) than conventional B mode (CEUS AUC 0.89 vs. B-mode US AUC 0.78), and diagnostic accuracy was also significantly higher with Kupffer-phase imaging (92\%) than with conventional B-mode imaging (74\%). The sensitivity, specificity, PPV, NPV, and accuracy of US studies cited in this review were summarized in Table 1 except for not available reports.

\section{PREDICTION OF MVI USING CONTRAST CT}

Contrast multi-detector low CT is commonly used for the definite diagnostic imaging of HCC. The diagnostic information obtained by contrast CT is tumor vascularity and morphology. There are some reports about the correlation between histologic differentiation and tumor vascularity. For example, Asayama et al. ${ }^{[22]}$ indicated that the arterial blood supply decreases significantly in poorly differentiated HCCs compared to moderately differentiated HCCs. Sanada et al. ${ }^{[23]}$ demonstrated that small HCCs intermingled with hypovascular areas and hypervascular areas in the arterial phase of contrast CT included poorly differentiated HCC components. Kawamura et al. ${ }^{[24]}$ also reported that heterogeneous enhancement with irregular ring-like structures in the arterial phase was a significant independent predictor of poorly differentiated HCC. On the other hand, fast tumor enhancement washout is also associated with poorly differentiated HCC. Nishie et al. ${ }^{[25]}$ indicated that poorly differentiated HCCs show faster tumor enhancement washout on contrast CT than non-poorly differentiated HCCs. However, Nakachi et al. ${ }^{[26]}$ demonstrated that the diagnostic accuracy for poorly differentiated HCC using tumor enhancement washout in the venous phase was low compared with heterogenous tumor enhancement in the arterial phase. They showed that sensitivity, specificity, PPV, NPV, and accuracy for predicting poor differentiation in small HCCs (up to $3 \mathrm{~cm}$ in diameter) by heterogenous 
Table 1. Studies with ultrasound for predicting poorly differentiation, non-single nodular type, or microvascular invasion

\begin{tabular}{|c|c|c|c|c|c|c|c|c|}
\hline Ref. & Modalities & Findings & Prediction & Sensitivity & Specificity & PPV & NPV & Accuracy \\
\hline Moribata et al. ${ }^{[10]}$ & B-mode US & Irregular or unclear margin & Poorly diff. & $89 \%$ & $67 \%$ & $19 \%$ & $99 \%$ & $69 \%$ \\
\hline Nuta et $a .^{[21]}$ & B-mode US & Irregular or unclear margin & Non-SN type & $72 \%$ & $85 \%$ & $96 \%$ & $39 \%$ & $74 \%$ \\
\hline Sugimoto et al.. ${ }^{[11]}$ & CEUS & Dead wood pattern & Poorly diff. & $80 \%$ & $96 \%$ & $86 \%$ & $94 \%$ & $92 \%$ \\
\hline Tanaka et al. ${ }^{[12]}$ & CEUS & Grade 4 (hypo-irregular) & Poorly diff. & $100 \%$ & $91 \%$ & $50 \%$ & $100 \%$ & $92 \%$ \\
\hline Tanaka et al..$^{[12]}$ & CEUS & Grade 4 (hypo-irregular) & MVI & $40 \%$ & $92 \%$ & $67 \%$ & $80 \%$ & $78 \%$ \\
\hline Feng et al. ${ }^{[17]}$ & CEUS & Washout time $<40 \mathrm{~s}$ & Poorly diff. & $24 \%$ & $97 \%$ & $65 \%$ & $61 \%$ & $69 \%$ \\
\hline Hatanaka et al. ${ }^{[18]}$ & CEUS & Irregular defect on Kupffer phase & Non-SN type & $80 \%$ & $96 \%$ & $92 \%$ & $89 \%$ & $90 \%$ \\
\hline Tada et al. ${ }^{[19]}$ & CEUS & Irregular defect on Kupffer phase & Non-SN type & $87 \%$ & $93 \%$ & $91 \%$ & $84 \%$ & $95 \%$ \\
\hline Nuta et al. ${ }^{[21]}$ & CEUS & Irregular defect on Kupffer phase & Non-SN type & $93 \%$ & $85 \%$ & $97 \%$ & $73 \%$ & $92 \%$ \\
\hline
\end{tabular}

PPV: positive predictive value; NPV: negative predictive value; US: ultrasound; SN: single nodular; CEUS: contrast enhanced ultrasonography; MVI: microvascular invasion

tumor enhancement were $75 \%, 90 \%, 48 \%, 97 \%$ and $88 \%$, respectively ${ }^{[26]}$. Accordingly, heterogenous tumor enhancement even in small HCC is an important observation for predicting HCC with poorly differentiated components.

Some reports show that irregular tumor margin in the venous phase of contrast CT is an important finding for predicting MVI or tumor differentiation. Lee et al. ${ }^{[27]}$ demonstrated that the presence of intra-tumoral vessels and aneurysms, tumor necrosis, attenuation of pre-contrast, the relative timing of washout, intratumoral attenuation heterogeneity, tumor margin, and tumor size were correlated with the pathological differentiation of HCC. In particular, the presence of intra-tumoral aneurysm was a highly specific finding for poorly differentiated HCC. Chou et al. ${ }^{[28]}$ showed that the sensitivity, specificity, PPV, NPV, and accuracy of the irregular tumor margin in predicting MVI in their retrospective study were $66 \%, 86.5 \%, 82.5 \%, 72.6 \%$ and $76.5 \%$, respectively, and those in their prospective study were $81.7 \%, 88.1 \%, 90.7 \%, 77.1 \%$ and $84.3 \%$, respectively ${ }^{[29]}$. Reginelli et al ${ }^{[30]}$ indicated that irregularity in tumor margins, as well as defects of peritumoral capsule are the most significant characteristics predicting MVI in HCC. Wu et al.$^{[31]}$ reported that irregular tumor margin was alone independent predictive factor for MVI among previously proposed predicting factors such as fluorine-18 fluorodeoxyglucose-positron emission tomography (FDG-PET) results and serum tumor markers. $\mathrm{Hu}$ et al. ${ }^{[32]}$ demonstrated in a meta-analysis that CT is superior to MRI in evaluating an irregular tumor margin for MVI assessment. Banerjee et al. ${ }^{[33]}$ showed new features of contrast CT that can also accurately predict histological MVI in HCC surgical candidates. These features include: the positivity of radiogemic venous invasion consisting of three separate imaging features; the persistence of discrete arterial tumor enhancement in the venous phase; partial or complete absence of hypodense halo; and absence of tumor-liver difference in the absence of a halo. Zhao et al. ${ }^{[34]}$ demonstrated that the predictive scoring model based on intra-tumoral arteries, non-nodular type of HCC, and absence of the radiological tumor capsule on preoperative CECT is of great value in the prediction of MVI regardless of tumor size. The sensitivity, specificity, PPV, NPV, and accuracy of CT studies cited in this review were summarized in Table 2 except for not available reports.

\section{PREDICTION OF MVI USING MRI}

The signal intensity of MRI can also be used to distinguish well differentiated HCC from moderately/poorly differentiated $\mathrm{HCC}^{[35-37]}$. For example, typical moderately/poorly differentiated HCC show hypointensity on T1-weighted imaging and hyperintensity on T2-weighted imaging. Enomoto et al. ${ }^{[38]}$ reported that hypointensity of tumor on T1-weighted imaging and tumor stain washout during the portal phase of dynamic MRI reflected poorer histological differentiation of HCCs, and the sensitivity, specificity, and the accuracy for diagnosis of poorly differentiated HCC using combined findings of hypointensity on T1-weighted imaging and tumor enhancement washout during the portal phase were $88 \%, 67 \%$ and $71 \%$, respectively. On the contrary, most of well-differentiated HCCs (83\%) showed non-hypointensity on T1-weighted image ${ }^{[38]}$. Min et al. ${ }^{[39]}$ 
Table 2. Studies with contrast enhanced computed tomography for predicting poorly differentiation, non-single nodular type, or microvascular invasion

\begin{tabular}{|c|c|c|c|c|c|c|c|c|}
\hline Ref. & Modalities & Findings & Prediction & Sensitivity & Specificity & PPV & NPV & Accuracy \\
\hline Nishie et $a l^{[25]}$ & CECT & Washout on portal-venous phase & Poorly diff. & $63 \%$ & $72 \%$ & $38 \%$ & $88 \%$ & $70 \%$ \\
\hline Nakachi et al. ${ }^{[26]}$ & CECT & Enhancement with non-enhanced area & Poorly diff. & $75 \%$ & $90 \%$ & $48 \%$ & $97 \%$ & $88 \%$ \\
\hline Nakachi et al. ${ }^{[26]}$ & CECT & Washout on portal-venous phase & Poorly diff. & $100 \%$ & $55 \%$ & $22 \%$ & $100 \%$ & $60 \%$ \\
\hline Nakachi et al. ${ }^{[26]}$ & CECT & Above combination & Poorly diff. & $75 \%$ & $92 \%$ & $55 \%$ & $97 \%$ & $90 \%$ \\
\hline Lee et $a / .{ }^{[27]}$ & CECT & Intra-tumoral aneurysm & Poorly diff. & $18 \%$ & $99 \%$ & $93 \%$ & $77 \%$ & $78 \%$ \\
\hline Lee et $\left.a\right|^{[27]}$ & CECT & Irregular tumor margin & Poorly diff. & $74 \%$ & $44 \%$ & $32 \%$ & $82 \%$ & $52 \%$ \\
\hline Chou et al. ${ }^{[28]}$ & CECT & Irregular tumor margin (retrospective) & $\mathrm{MVI}$ & $66 \%$ & $87 \%$ & $83 \%$ & $73 \%$ & $77 \%$ \\
\hline Chou et al. ${ }^{[28]}$ & CECT & Irregular tumor margin (prospective) & MVI & $82 \%$ & $87 \%$ & $91 \%$ & $77 \%$ & $84 \%$ \\
\hline Wu et al. ${ }^{[31]}$ & CECT & Irregular tumor margin & MVI & $87 \%$ & $73 \%$ & $43 \%$ & $96 \%$ & $76 \%$ \\
\hline Reginelli et al. ${ }^{[30]}$ & СECT & Irregular tumor margin & $\mathrm{MVI}$ & $66 \%$ & $94 \%$ & $84 \%$ & $86 \%$ & $85 \%$ \\
\hline Reginelli et al. ${ }^{[30]}$ & CECT & Incomplete peritumoral capsule & MVI & $81 \%$ & $90 \%$ & $76 \%$ & $91 \%$ & $89 \%$ \\
\hline Banerjee et al. ${ }^{[33]}$ & CECT & Positivity of radiogemic venous invasion & MVI & $76 \%$ & $94 \%$ & $83 \%$ & $91 \%$ & $89 \%$ \\
\hline Zhao et al. ${ }^{[34]}$ & CECT & Score model (validation cohort) & MVI & $82 \%$ & $83 \%$ & $74 \%$ & $88 \%$ & N/A \\
\hline
\end{tabular}

PPV: positive predictive value; NPV: negative predictive value; CECT: contrast enhanced computed tomography; MVI: microvascular invasion; N/A: not available

reported that intra-tumoral fat detected by chemical-shift of T1-weighted image indicates lower risk for MVI of HCC.

The recent advances in MRI instrumentation has allowed high quality diffusion weighted images (DWI) to be obtained. The correlation between the apparent diffusion coefficient (ADC) values on DWI and histologic differentiation have been reported ${ }^{[40-44]}$, suggesting that low ADC values can be a useful predictor of $\mathrm{MVI}^{[45-47]}$. However, there was no notable threshold of ADC value for predicting poorly differentiated HCC on metaanalysis ${ }^{[48]}$. Park et al. ${ }^{[49]}$ showed that hypervascular HCCs with low ADC value could be interpreted as poorly differentiated HCCs, while it was difficult to differentiate between well- and poorly differentiated HCCs that are hypovascular. Among all ADC parameters, Moriya et al. ${ }^{[50]}$ demonstrated that the minimum $\mathrm{ADC}$ value was the most useful in distinguishing poorly differentiated HCC in $3 \mathrm{D}$ analysis of ADC histograms. On the other hand, Ogihara et al. ${ }^{[51]}$ indicated that contrast-to-noise ratio (CNR) between the lesion and the liver parenchyma on DWI might be more useful than the ADC values for predicting poorly differentiated HCCs. Iwasa et al. ${ }^{[52]}$ also indicated that DWI CNR and the lesion-to-liver relative contrast ratio (RCR) on DWI are superior in predicting histologic differentiation than the ADC values, T2-weighted RCR, and ethoxybenzyl-hepatobiliary RCR. Mori et al ${ }^{[53]}$ showed the usefulness of ADC mapping in predicting preoperative malignant potential of HCC. On the basis of their data, the sensitivity, specificity, PPV, NPV, and accuracy for predicting poorly differentiated HCC is $93 \%, 68 \%, 54 \%, 96 \%$ and $75 \%$, respectively, and those for predicting MVI is $89 \%, 58 \%, 31 \%, 96 \%$ and $63 \%$, respectively. They suggested that hypointense HCC on ADC mapping are characterized by poor histological differentiation and more frequent microscopic portal inva$\operatorname{sion}^{[53]}$. Zhao et al ${ }^{[54]}$ showed the usefulness of the combination of the true diffusion coefficient value and an irregular shape on hepatobiliary phase for predicting MVI, and the sensitivity and specificity were improved to $94.4 \%$ and $63.6 \%$ respectively. Wang et al ${ }^{[55]}$ reported that other diffusion parameters, such as mean kurtosis value on diffusion kurtosis imaging, and irregular circumferential enhancement on dynamic MRI were independent risk factors for MVI of HCC. The combination of higher mean kurtosis values and irregular shape are potential predictive biomarkers for $\mathrm{MVI}^{[55]}$.

Gadolinium-ethoxybenzyl diethylenetriamine penta-acetic acid-enhanced magnetic resonance imaging (EOB-MRI) is now commonly used for the diagnosis of HCC. With its use, there have been increasing reports of predicting MVI using dynamic MRI including hepatobiliary phase of EOB-MRI. Chang et al. ${ }^{[56]}$ indicated that relatively low arterial enhancement on arterial phase of EOB-MRI and low ADC value were predictive of worse histological grades of HCC. Kim et al. ${ }^{[57]}$ suggested focusing on the peritumoral hypointensity on hepatobiliary phase of EOB-MRI for predicting MVI. The sensitivity, specificity, PPV and NPV 
Table 3. Studies with magnetic resonance imaging for predicting poorly differentiation, non-single nodular type, or microvascular invasion

\begin{tabular}{|c|c|c|c|c|c|c|c|c|}
\hline Ref. & Modalities & Findings & Prediction & Sensitivity & Specificity & PPV & NPV & Accuracy \\
\hline Enomoto et al. ${ }^{[38]}$ & $\begin{array}{l}\text { Plain + dynamic } \\
\text { MRI }\end{array}$ & $\begin{array}{l}\text { Hypointensity on T1-weighted } \\
\text { imaging and washout on portal- } \\
\text { venous phase }\end{array}$ & Poorly diff. & $88 \%$ & $67 \%$ & $\mathrm{~N} / \mathrm{A}$ & $\mathrm{N} / \mathrm{A}$ & $71 \%$ \\
\hline Mori et al. ${ }^{[53]}$ & Plain MRI & Hypointensity on ADC map & Poorly diff. & $93 \%$ & $68 \%$ & $54 \%$ & $96 \%$ & $75 \%$ \\
\hline Mori et al. ${ }^{[53]}$ & Plain MRI & Hypointensity on ADC map & MVI & $89 \%$ & $58 \%$ & $31 \%$ & $96 \%$ & $63 \%$ \\
\hline Wang et al. ${ }^{[55]}$ & Plain MRI & Mean kurtosis values $>0.917$ & MVI & $70 \%$ & $77 \%$ & $70 \%$ & $77 \%$ & $74 \%$ \\
\hline Kim et al. ${ }^{[57]}$ & EOB-MRI & Peritumoral hypointensity on HBP & MVI & $38 \%$ & $93 \%$ & $89 \%$ & $53 \%$ & $62 \%$ \\
\hline Zhao et $a l^{[54]}$ & EOB-MRI & Irregular tumor margin & MVI & $50 \%$ & $88 \%$ & $69 \%$ & $76 \%$ & $75 \%$ \\
\hline Lee et $a{ }^{[58]}$ & EOB-MRI & Arterial peritumoral enhancement & MVI & $54 \%$ & $88 \%$ & $68 \%$ & $80 \%$ & $77 \%$ \\
\hline Lee et $a ._{.[58]}$ & EOB-MRI & Irregular tumor margin & MVI & $70 \%$ & $69 \%$ & $51 \%$ & $83 \%$ & $69 \%$ \\
\hline Lee et $a ._{.}^{[58]}$ & EOB-MRI & Peritumoral hypointensity on HBP & MVI & $32 \%$ & $92 \%$ & $65 \%$ & $74 \%$ & $73 \%$ \\
\hline Tada et $a l^{[60]}$ & EOB-MRI & Irregular tumor margin & Non-SN type & $97 \%$ & $72 \%$ & $74 \%$ & $97 \%$ & $83 \%$ \\
\hline Chen et al. ${ }^{[61]}$ & EOB-MRI & Irregular tumor margin & Non-SN type & $96 \%$ & $79 \%$ & $87 \%$ & $94 \%$ & $89 \%$ \\
\hline Kobayashi et al. ${ }^{[62]}$ & EOB-MRI & Irregular tumor margin & Non-SN type & $64 \%$ & $96 \%$ & $93 \%$ & $77 \%$ & $81 \%$ \\
\hline Chen et al. ${ }^{[61]}$ & $E O B-M R I+C E C T$ & Irregular tumor margin & Non-SN type & $98 \%$ & $84 \%$ & $90 \%$ & $94 \%$ & $93 \%$ \\
\hline Kobayashi et al. ${ }^{[62]}$ & EOB-MRI + CEUS & Irregular tumor margin & Non-SN type & $85 \%$ & $95 \%$ & $94 \%$ & $88 \%$ & $91 \%$ \\
\hline
\end{tabular}

PPV: positive predictive value; NPV: negative predictive value; MRI: magnetic resonance imaging; N/A: not available; ADC: apparent diffusion coefficient; MVI: microvascular invasion; EOB-MRI: Gadolinium-ethoxybenzyl diethylenetriamine pentaacetic acid-enhanced magnetic resonance imaging; HBP: hepatobiliary phase; SN: single nodular; CECT: contrast enhanced computed tomography; CEUS: contrast enhanced ultrasonography

were $38.3 \%, 93.2 \%, 88.5 \%, 52.6 \%$ and $62 \%$ respectively. Lee et al ${ }^{[58]}$ also demonstrated that a combination of two or more of the following; arterial peritumoral enhancement, irregular tumor margin, and peritumoral hypointensity on hepatobiliary phase, can be used as a preoperative imaging biomarker for predicting MVI, with specificity $>90 \%$. Hu et al. ${ }^{[59]}$ also reported in a systemic review and meta-analysis that peritumoral enhancement and peritumoral hypointensity on hepatobiliary phase were highly specific (90\%-94\%) but low sensitive findings (29\%-40\%) for predicting MVI.

On distinguishing between the SN type and non-SN type using EOB-MRI, Tada et al. ${ }^{[60]}$ demonstrated that the sensitivity, specificity, and accuracy of EOB-MRI for identifying non-SN were equal to or higher than that using angiography-assisted CT. Chen et al. ${ }^{[61]}$ also compared the diagnostic ability of EOB-MRI and contrast CT. The sensitivities, specificities, and accuracies for the diagnosis of non-SN type were $71.4 \%$, $81.6 \%$, and $75.5 \%$ in contrast CT, $96.4 \%, 78.9 \%$, and $89.3 \%$ in EOB-MRI, and $98.2 \%, 84.2 \%$, and $92.5 \%$ in combination, respectively. They concluded that contrast CT combined with EOB-MRI offers a more accurate imaging evaluation for HCC macroscopic classification than either modality alone ${ }^{[61]}$. Kobayashi et al. ${ }^{[62]}$ compared the ability of EOB-MRI and CEUS to predict macroscopic type, and found that the sensitivity, specificity, PPV, NPV, and accuracy for the diagnosis of non-SN type were $64.1 \%, 95.7 \%, 92.6 \%, 76.9 \%$ and 81.2\% in EOB-MRI, 56.4\%, 97.8\%, 95.7\%, 72.6\% and 78.8\% in CEUS, and $84.6 \%, 95.7 \%, 94.3 \%, 88 \%$ and $90.6 \%$ in combination, respectively. The combined diagnosis of EOB-MRI and CEUS provides highest diagnostic ability $^{[62]}$. Iwamoto et al. ${ }^{[63]}$ also showed that the diagnostic ability for macroscopic classification of nodular HCC of the post-vascular phase of CEUS with Sonazoid was comparable with that of hepatobiliary phase of EOB-MRI, and the combination of the two modalities provided a more accurate diagnostic performance. The sensitivity, specificity, PPV, NPV, and accuracy of MRI studies cited in this review were summarized in Table 3 except for not available reports.

\section{DISCUSSION}

This article reviews the current status of predicting MVI using common imaging modalities for the diagnosis of HCC. MVI is strongly associated with histologic differentiation and macroscopic type. Poorly differentiated HCCs are characterized by hypovascular components and faster tumor enhancement washout on dynamic imaging. Non-SN type HCCs are characterized by irregular shape image. The possible mechanism 
which could interpret the correlation between imaging features and probability of MVI is that HCC has a strong tendency of invasive growth along with de-differentiation from well to poorly differentiated HCC. Therefore, the accurate diagnosis of histologic differentiation and macroscopic type is essential for accurate prediction of MVI.

US including CEUS is the most non-invasive among imaging modalities. Although most of the previous reports cited in "US" section were about the diagnosis of HCC with poor differentiation or non-SN type, which suggested that direct connection between US and diagnosis of MVI was a few, useful US parameters for predicting poorly differentiated HCC or MVI are irregular intra-tumoral artery, fast washout of tumor enhancement, and irregular tumor margin. MFI should be used to assess the intra-tumoral angioarchitecture, with the deadwood pattern being a highly specific finding for predicting MVI. Although the optimal cut off time is yet unknown, shorter washout time of tumor enhancement is also a specific finding. Based on previous reports on assessing tumor shape by imaging, post-vascular phase (Kupffer phase) of CEUS is considered more accurate than B-mode US or contrast CT, and the diagnostic ability of CEUS for predicting HCC macroscopic type would be equal to that of the hepatobiliary phase of EOB-MRI. However, US has several disadvantages, such as poor visualization due to dead space, artifacts, and deep lesion, and difficulty of whole scan in larger tumors. Therefore, when the whole tumor cannot be scanned by US, another imaging assessment using EOB-MRI or contrast CT is necessary.

Factors to consider in predicting poorly differentiated HCC or MVI on contrast CT are heterogenous enhancement including hypovascular components, fast washout of tumor enhancement, complete or partial absence of peritumoral capsule (halo), the presence of intra-tumoral vessels and aneurysms on venous phase, and irregular tumor margin. Although complete or partial absence of peritumoral capsule, the presence of intra-tumoral vessels and aneurysms, and irregular tumor margin can be easily evaluated in large HCC, these parameters are difficult to assess in small HCCs. The heterogenous enhancement and fast tumor enhancement washout are useful findings in predicting poor histologic differentiation of small HCCs.

Tumor tissue characterization such as water, fat, and metal content, or diffusion of water molecules can be easily obtained by plain MRI. As non-hypointense HCC on T1-weighted image reflects well differentiation of HCC, the MVI risk would be low. Since intra-tumoral fat is also often seen in well differentiated HCCs, HCCs containing fat components would be at low risk of MVI. Accordingly, T1-weighted image including chemical shift is important in predicting low risk of MVI. Previous reports also demonstrated that low ADC value reflects poor differentiation. However, there is no adequate cut-off value to distinguish poorly and nonpoorly differentiated HCC on meta-analysis. This may be because the absolute ADC value depends on the MRI equipment coil systems, imagers, vendors, and field strengths ${ }^{[64]}$. Since the contrast between tumor and adjacent liver tissue, measured by CNR, and the RCR on DWI, are superior in predicting poor differentiation compared to the ADC values, the assessment of tumor contrast to adjacent liver tissue on DWI or ADC map should be more appropriate and universal in clinical practice than quantification of ADC values. As such, care should be taken when evaluating a very high intense HCC on DWI or low intense HCC on ADC map. When directly predicting MVI using EOB-MRI, the important parameters include irregular margin, arterial peritumoral enhancement (relative hypovascularity), and peritumoral hypointensity on hepatobiliary phase. Although the specificities of these findings for predicting MVI were very high, the sensitivities were low. Therefore, attention should be paid for the false negativity of these findings. To improve the sensitivity for predicting MVI, the combined evaluation with plain MRI including DWI and EOB-MRI may be useful, because the evaluation of diffusion parameters is highly sensitive for predicting poor differentiation or MVI. Among common imaging modalities, the most information for predicting MVI can be obtained from MRI, Furthermore, there are some reports that the combination of EOB-MRI with CECT or CEUS improved the accuracy in predicting non-SN type. As mentioned above, combination of MRI with other imaging modalities may provide a more accurate assessment of malignant potential of HCC. 
As other tumor factors related to MVI, tumor markers ${ }^{[65,66]}$ and FDG-PET uptake ${ }^{[67]}$ have been reported. As FDG-PET is not used as common imaging modality for the diagnosis of HCC, the papers about the predictability of FDG-PET for MVI were omitted from this review. Although tumor markers such as alpha-fetoprotein and des- $\gamma$-carboxy prothrombin (DCP) are closely related to the presence of MVI, they are also highly expressed in patients with benign diseases such as chronic hepatitis and liver cirrhosis ${ }^{[68]}$. Furthermore, as several cut-off values according to studies were suggested, the best cut-off value has not been unknown. However, Shirabe et al ${ }^{[69]}$ reported that a scoring system for predicting MVI using tumor size, serum DCP levels, and FDG-PET uptake can provide a precise prediction of MVI, and the sensitivity and specificity were $100 \%$ and $90.9 \%$, respectively. Probably, tumor marker levels would be helpful for predicting MVI.

There are some limitations in this research field. Firstly, although some highly specific or sensitive imaging findings for predicting poorly differentiated HCC or MVI have been reported, there are no highly accurate diagnostic findings. This may be due to limited accuracy of identifying histologic differentiation or MVI from resected specimens. MVI would often be missed if thorough microscopic examination is not performed, and histologic differentiation would be judged as non-poorly differentiated HCC if poorly differentiated components are not dominant. It is difficult to search for MVI throughout the whole tumor using a microscope, and MVI detection depends on the serial slice width of the tumor specimen. The thinner the specimen, the more accurate the MVI detection. Secondly, since most of previous reports are smallcohort, single-center, and retrospective, and diagnostic ability also depends on the performance of imaging equipment, their conclusions might be unreliable and biased. To validate their results, large scale prospective studies are needed. Lastly, adequate treatment strategy based on MVI prediction or histologic differentiation has not been established. At this time, if a HCC patient is predicted to have high risk of MVI as assessed by imaging, it should be treated as advanced HCC even after resection or local ablation.

In conclusion, HCCs with high grade malignant potential can be diagnosed with commonly used imaging modalities. For accurate prediction of MVI in HCC, the diagnosis of poor histologic differentiation or non$\mathrm{SN}$ type is needed, and the combination of MRI with other imaging modalities should be used.

\section{DECLARATIONS}

\section{Authors' contributions}

The author contributed solely to the article.

\section{Availability of data and materials}

Not applicable.

\section{Financial support and sponsorship}

None.

\section{Conflicts of interest}

All authors declared that there are no conflicts of interest.

\section{Ethical approval and consent to participate}

Not applicable.

\section{Consent for publication}

Not applicable.

\section{Copyright}

(c) The Author(s) 2018. 


\section{REFERENCES}

1. Yuki K, Hirohashi S, Sakamoto M, Kanai T, Shimosato Y. Growth and spread of hepatocellular carcinoma. A review of 240 consecutive autopsy cases. Cancer 1990;66:2174-9.

2. Kudo M. Multistep human hepatocarcinogenesis: correlation of imaging with pathology. J Gastroenterol 2009;44 Suppl 19:112-8.

3. Fukuda S, Itamoto T, Nakahara H, Kohashi T, Ohdan H, et al. Clinicopathologic features and prognostic factors of resected solitary smallsized hepatocellular carcinoma. Hepatogastroenterology 2005;52:1163-7.

4. Esnaola NF, Lauwers GY, Mirza NQ, Nagorney DM, Doherty D, et al. Predictors of microvascular invasion in patients with hepatocellular carcinoma who are candidates for orthotopic liver transplantation. J Gastrointest Surg 2002;6:224-32.

5. Iguchi T, Aishima S, Sanefuji K, Fujita N, Sugimachi K, et al. Both fibrous capsule formation and extracapsular penetration are powerful predictors of poor survival in human hepatocellular carcinoma: a histological assessment of 365 patients in Japan. Ann Surg Oncol 2009;16:2539-46.

6. Kanai T, Hirohashi S, Upton MP, Noguchi M, Kishi K, et al. Pathology of small hepatocellular carcinoma. A proposal for a new gross classification. Cancer 1987;60:810-9.

7. Hui AM, Takayama T, Sano K, Kubota K, Akahane M, et al. Predictive value of gross classification of hepatocellular carcinoma on recurrence and survival after hepatectomy. J Hepatol 2000;33:975-9.

8. Shimada M, Rikimaru T, Hamatsu T, Yamashita Y, Terashi T, et al. The role of macroscopic classification in nodular-type hepatocellular carcinoma. Am J Surg 2001;182:177-82.

9. Nakashima Y, Nakashima O, Tanaka M, Okuda K, Nakashima M, et al. Portal vein invasion and intrahepatic micrometastasis in small hepatocellular carcinoma by gross type. Hepatol Res 2003;26:142-7.

10. Moribata K, Tamai H, Shingaki N, Mori Y, Enomoto S, et al. Assessment of malignant potential of small hypervascular hepatocellular carcinoma using B-mode ultrasonography. Hepatol Res 2011;41:233-9.

11. Sugimoto K, Moriyasu F, Kamiyama N, Metoki R, Yamada M, et al. Analysis of morphological vascular changes of hepatocellular carcinoma by microflow imaging using contrast-enhanced sonography. Hepatol Res 2008;38:790-9.

12. Tanaka H, lijima H, Higashiura A, Yoh K, Ishii A, et al. New malignant grading system for hepatocellular carcinoma using the Sonazoid contrast agent for ultrasonography. J Gastroenterol 2014;49:755-63.

13. Xia Y, Jiang YX, Dai Q, Xiao Y, Lv K, et al. Contrast-enhanced ultrasound of hepatocellular carcinoma: correlation of washout time and angiogenesis. Clin Hemorheol Microcirc 2011;48:265-73.

14. Xu JF, Liu HY, Shi Y, Wei ZH, Wu Y. Evaluation of hepatocellular carcinoma by contrast-enhanced sonography: correlation with pathologic differentiation. J Ultrasound Med 2011;30:625-33.

15. Pei XQ, Liu LZ, Liu M, Zheng W, Han F, et al. Contrast-enhanced ultrasonography of hepatocellular carcinoma: correlation between quantitative parameters and histological grading. Br J Radiol 2012;85:e740-7.

16. Loria F, Loria G, Basile S, Crea G, Randazzo D, et al. Contrast-enhanced ultrasound of hepatocellular carcinoma: correlation between enhancement pattern and cellular differentiation on histopathlogy. Updates Surg 2012;64:247-55.

17. Feng Y, Qin XC, Luo Y, Li YZ, Zhou X. Efficacy of contrast-enhanced ultrasound washout rate in predicting hepatocellular carcinoma differentiation. Ultrasound Med Biol 2015;41:1553-60.

18. Hatanaka K, Chung H, Kudo M, Haji S, Minami Y, et al. Usefulness of the post-vascular phase of contrast-enhanced ultrasonography with sonazoid in the evaluation of gross types of hepatocellular carcinoma. Oncology 2010;78 Suppl 1:53-9.

19. Tada T, Kumada T, Toyoda H, Ito T, Sone Y, et al. Utility of contrast-enhanced ultrasound with perflubutane for diagnosing the macroscopic type of small nodular hepatocellular carcinomas. Eur Radiol 2014;24:2157-66.

20. Hatanaka K, Minami Y, Kudo M, Inoue T, Chung H, et al. The gross classification of hepatocellular carcinoma: usefulness of contrastenhanced US. J Clin Ultrasound 2014;42:1-8.

21. Nuta J, Shingaki N, Ida Y, Shimizu R, Hayami S, et al. Irregular defects in hepatocellular carcinomas during the Kupffer phase of contrastenhanced ultrasonography with perfluorobutane microbubbles: pathological features and metastatic recurrence after surgical resection. Ultrasound Med Biol 2017;43:1829-36.

22. Asayama Y, Yoshimitsu K, Nishihara Y, Irie H, Aishima S, et al. Arterial blood supply of hepatocellular carcinoma and histologic grading: radiologic-pathologic correlation. AJR Am J Roentgenol 2008;190:W28-34.

23. Sanada Y, Yoshida K, Itoh H. Comparison of CT enhancement patterns and histologic features in hepatocellular carcinoma up to $2 \mathrm{~cm}$ : assessment of malignant potential with claudin-10 immunohistochemistry. Oncol Rep 2007;17:1177-82.

24. Kawamura Y, Ikeda K, Hirakawa M, Yatsuji H, Sezaki H, et al. New classification of dynamic computed tomography images predictive of malignant characteristics of hepatocellular carcinoma. Hepatol Res 2010;40:1006-14.

25. Nishie A, Yoshimitsu K, Okamoto D, Tajima T, Asayama Y, et al. CT prediction of histological grade of hypervascular hepatocellular carcinoma: utility of the portal phase. Jpn J Radiol 2013;31:89-98.

26. Nakachi K, Tamai H, Mori Y, Shingaki N, Moribata K, et al. Prediction of poorly differentiated hepatocellular carcinoma using contrast computed tomography. Cancer Imaging 2014;14:7.

27. Lee JH, Lee JM, Kim SJ, Baek JH, Yun SH, et al. Enhancement patterns of hepatocellular carcinomas on multiphasicmultidetector row CT: comparison with pathological differentiation. Br J Radiol 2012;85:e573-83.

28. Chou CT, Chen RC, Lee CW, Ko CJ, Wu HK, et al. Prediction of microvascular invasion of hepatocellular carcinoma by pre-operative CT imaging. Br J Radiol 2012;85:778-83.

29. Chou CT, Chen RC, Lin WC, Ko CJ, Chen CB, et al. Prediction of microvascular invasion of hepatocellular carcinoma: preoperative CT and histopathologic correlation. AJR Am J Roentgenol 2014;203:W253-9.

30. Reginelli A, Vanzulli A, Sgrazzutti C, Caschera L, Serra N, et al. Vascular microinvasion from hepatocellular carcinoma: CT findings and pathologic correlation for the best therapeutic strategies. Med Oncol 2017;34:93. 
31. Wu TH, Hatano E, Yamanaka K, Seo S, Taura K, et al. A non-smooth tumor margin on preoperative imaging predicts microvascular invasion of hepatocellular carcinoma. Surg Today 2016;46:1275-81.

32. Hu H, Zheng Q, Huang Y, Huang XW, Lai ZC, et al. A non-smooth tumor margin on preoperative imaging assesses microvascular invasion of hepatocellular carcinoma: a systematic review and meta-analysis. Sci Rep 2017;7:15375.

33. Banerjee S, Wang DS, Kim HJ, Sirlin CB, Chan MG, et al. A computed tomography radiogenomic biomarker predicts microvascular invasion and clinical outcomes in hepatocellular carcinoma. Hepatology 2015;62:792-800.

34. Zhao H, Hua Y, Dai T, He J, Tang M, et al. Development and validation of a novel predictive scoring model for microvascular invasion in patients with hepatocellular carcinoma. Eur J Radiol 2017;88:32-40.

35. Muramatsu Y, Nawano S, Takayasu K, Moriyama N, Yamada T, et al. Early hepatocellular carcinoma: MR imaging. Radiology 1991;181:209-13.

36. Kadoya M, Matsui O, Takashima T, Nonomura A. Hepatocellular carcinoma: correlation of MR imaging and histopathologic findings. Radiology 1992;183:819-25.

37. Ebara M, Fukuda H, Kojima Y, Morimoto N, Yoshikawa M, et al. Small hepatocellular carcinoma: relationship of signal intensity to histopathologic findings and metal content of the tumor and surrounding hepatic parenchyma. Radiology 1999;210:81-8.

38. Enomoto S, Tamai H, Shingaki N, Mori Y, Moribata K, et al. Assessment of hepatocellular carcinomas using conventional magnetic resonance imaging correlated with histological differentiation and a serum marker of poor prognosis. Hepatol Int 2011;5:730-7.

39. Min JH, Kim YK, Lim S, Jeong WK, Choi D, et al. Prediction of microvascular invasion of hepatocellular carcinomas with gadoxetic acidenhanced MR imaging: impact of intra-tumoral fat detected on chemical-shift images. Eur J Radiol 2015;84:1036-43.

40. Heo SH, Jeong YY, Shin SS, Kim JW, Lim HS, et al. Apparent diffusion coefficient value of diffusion-weighted imaging for hepatocellular carcinoma: correlation with the histologic differentiation and the expression of vascular endothelial growth factor. Korean J Radiol 2010;11:295-303.

41. Nishie A, Tajima T, Asayama Y, Ishigami K, Kakihara D, et al. Diagnostic performance of apparent diffusion coefficient for predicting histological grade of hepatocellular carcinoma. Eur J Radiol 2011;80:e29-33.

42. Nakanishi M, Chuma M, Hige S, Omatsu T, Yokoo H, et al. Relationship between diffusion-weighted magnetic resonance imaging and histological tumor grading of hepatocellular carcinoma. Ann Surg Oncol 2012;19:1302-9.

43. Tang Y, Wang H, Ma L, Zhang X, Yu G, et al. Diffusion-weighted imaging of hepatocellular carcinomas: a retrospective analysis of correlation between apparent diffusion coefficients and histological grade. Abdom Radiol (NY) 2016;41:1539-45.

44. Li X, Zhang K, Shi Y, Wang F, Meng X. Correlations between the minimum and mean apparent diffusion coefficient values of hepatocellular carcinoma and tumor grade. J Magn Reson Imaging 2016;44:1442-7.

45. Xu P, Zeng M, Liu K, Shan Y, Xu C, et al. Microvascular invasion in small hepatocellular carcinoma: is it predictable with preoperative diffusion-weighted imaging? J Gastroenterol Hepatol 2014;29:330-6.

46. Suh YJ, Kim MJ, Choi JY, Park MS, Kim KW. Preoperative prediction of the microvascular invasion of hepatocellular carcinoma with diffusion-weighted imaging. Liver Transpl 2012;18:1171-8.

47. Okamura S, Sumie S, Tonan T, Nakano M, Satani M, et al. Diffusion-weighted magnetic resonance imaging predicts malignant potential in small hepatocellular carcinoma. Dig Liver Dis 2016;48:945-52.

48. Chen J, Wu M, Liu R, Li S, Gao R, et al. Preoperative evaluation of the histological grade of hepatocellular carcinoma with diffusionweighted imaging: a meta-analysis. PLoS One 2015;10:e0117661.

49. Park IK, Yu JS, Cho ES, Kim JH, Chung JJ. Apparent diffusion coefficient of hepatocellular carcinoma on diffusion-weighted imaging: histopathologic tumor grade versus arterial vascularity during dynamic magnetic resonance imaging. PLoS One 2018;13:e0197070.

50. Moriya T, Saito K, Tajima Y, Harada TL, Araki Y, et al. 3D analysis of apparent diffusion coefficient histograms in hepatocellular carcinoma: correlation with histological grade. Cancer Imaging 2017;17:1.

51. Ogihara Y, Kitazume Y, Iwasa Y, Taura S, Himeno Y, et al. Prediction of histological grade of hepatocellular carcinoma using quantitative diffusion-weighted MRI: a retrospective multivendor study. Br J Radiol 2018;91:20170728.

52. Iwasa Y, Kitazume Y, Tateishi U, Saida Y, Ban D, et al. Hepatocellular carcinoma histological grade prediction: a quantitative comparison of diffusion-weighted, T2-weighted, and hepatobiliary-phase magnetic resonance imaging. J Comput Assist Tomogr 2016;40:463-70.

53. Mori Y, Tamai H, Shingaki N, Moribata K, Deguchi H, et al. Signal intensity of small hepatocellular carcinoma on apparent diffusion coefficient mapping and outcome after radiofrequency ablation. Hepatol Res 2015;45:75-87.

54. Zhao W, Liu W, Liu H, Yi X, Hou J, et al. Preoperative prediction of microvascular invasion of hepatocellular carcinoma with IVIM diffusion-weighted MR imaging and Gd-EOB-DTPA-enhanced MR imaging. PLoS One 2018;13:e0197488.

55. Wang WT, Yang L, Yang ZX, Hu XX, Ding Y, et al. Assessment of microvascular invasion of hepatocellular carcinoma with diffusion kurtosis imaging. Radiology 2018;286:571-80.

56. Chang WC, Chen RC, Chou CT, Lin CY, Yu CY, et al. Histological grade of hepatocellular carcinoma correlates with arterial enhancement on gadoxetic acid-enhanced and diffusion-weighted MR images. Abdom Imaging 2014;39:1202-12.

57. Kim KA, Kim MJ, Jeon HM, Kim KS, Choi JS, et al. Prediction of microvascular invasion of hepatocellular carcinoma: usefulness of peritumoral hypointensity seen on gadoxetate disodium-enhanced hepatobiliary phase images. J Magn Reson Imaging 2012;35:629-34.

58. Lee S, Kim SH, Lee JE, Sinn DH, Park CK. Preoperative gadoxetic acid-enhanced MRI for predicting microvascular invasion in patients with single hepatocellular carcinoma. J Hepatol 2017;67:526-34.

59. Hu HT, Shen SL, Wang Z, Shan QY, Huang XW, et al. Peritumoral tissue on preoperative imaging reveals microvascular invasion in hepatocellular carcinoma: a systematic review and meta-analysis. Abdom Radiol (NY) 2018;43:3324-30.

60. Tada T, Kumada T, Toyoda H, Ito T, Sone Y, et al. Diagnostic accuracy for macroscopic classification of nodular hepatocellular carcinoma: comparison of gadolinium ethoxybenzyl diethylenetriamine pentaacetic acid-enhanced magnetic resonance imaging and angiographyassisted computed tomography. J Gastroenterol 2015;50:85-94.

61. Chen C, Zhao H, Fu X, Huang L, Tang M, et al. Contrast-enhanced computed tomography plus gadolinium-ethoxybenzyl diethylenetriamine 
pentaacetic acid-enhanced magnetic resonance imaging for gross classification of hepatocellular carcinoma. Oncotarget 2017;8:29741-50.

62. Kobayashi T, Aikata H, Hatooka M, Morio K, Morio R, et al. Usefulness of combining gadolinium-ethoxybenzyl-diethylenetriamine pentaacetic acid-enhanced magnetic resonance imaging and contrast-enhanced ultrasound for diagnosing the macroscopic classification of small hepatocellular carcinoma. Eur Radiol 2015;25:3272-81.

63. Iwamoto T, Imai Y, Kogita S, Igura T, Sawai Y, et al. Comparison of contrast-enhanced ultrasound and gadolinium-ethoxybenzyldiethylenetriamine pentaacetic acid-enhanced MRI for the diagnosis of macroscopic type of hepatocellular carcinoma. Dig Dis 2016;34:679-86.

64. Sasaki M, Yamada K, Watanabe Y, Matsui M, Ida M, et al. Variability in absolute apparent diffusion coefficient values across different platforms may be substantial: a multivendor, multi-institutional comparison study. Radiology 2008;249:624-30.

65. Hirokawa F, Hayashi M, Miyamoto Y, Asakuma M, Shimizu T, et al. Outcomes and predictors of microvascular invasion of solitary hepatocellular carcinoma. Hepatol Res 2014;44:846-53.

66. Masuda T, Beppu T, Okabe H, Nitta H, Imai K, et al. Predictive factors of pathological vascular invasion in hepatocellular carcinoma within $3 \mathrm{~cm}$ and three nodules without radiological vascular invasion. Hepatol Res 2016;46:985-91.

67. Kobayashi T, Aikata H, Honda F, Nakano N, Nakamura Y, et al. Preoperative fluorine 18 fluorodeoxyglucose positron emission tomography/ computed tomography for prediction of microvascular invasion in small hepatocellular carcinoma. J Comput Assist Tomogr 2016;40:524-30

68. Zhang X, Li J, Shen F, Lau WY. Significance of presence of microvascular invasion in specimens obtained after surgical treatment of hepatocellular carcinoma. J Gastroenterol Hepatol 2018;33:347-54.

69. Shirabe K, Toshima T, Kimura K, Yamashita Y, Ikeda T, et al. New scoring system for prediction of microvascular invasion in patients with hepatocellular carcinoma. Liver Int 2014;34:937-41. 